\title{
Éléments théoriques en agroécologie : l'intensivité écologique
}

\author{
Michel Griffon ${ }^{*}$ \\ AEI, Pour une agriculture écologiquement intensive, 55, rue Rabelais, 49000 Angers, France
}

Reçu le 19 décembre 2016 - Accepté le 21 mars 2017

\begin{abstract}
Résumé - L'agroécologie est la science qui traite des agroécosystèmes. L'intensification écologique est le processus par lequel on intensifie l'usage de fonctionnalités écologiques des agroécosystèmes afin d'obtenir une production plus abondante, n'atteignant pas l'environnement et respectant les lois de la viabilité écologique. On parle alors d'agriculture écologiquement intensive. L'intensivité écologique est un domaine de créativité technique importante mais qui manque encore de bases théoriques. Cet article propose un tour d'horizon de quelques éléments théoriques disponibles. Il apparaît que la gestion d'agroécosystèmes écologiquement intensifs demande des références transdisciplinaires nouvelles.
\end{abstract}

Mots clés : agroécologie / agriculture écologiquement intensive / agroécosystème / agriculture / discipline

Abstract - Theoretical elements in agroecology: ecological intensivity. Agroecology is the science of agroecosystems. Ecological intensification is the process by which ecological functionalities can be intensified to obtain higher biomass production from agroecosystems, environmentally friendly, and in a way that has to be compatible with ecological viability principles. This statement defines an ecologically intensive agriculture. Ecological intensification is a very creative domain for new technologies but theoretical bases are still lacking. This paper enumerates some available theoretical elements. It appears that the management of ecologically intensive agroecoystems needs new transdisciplinary approaches.

Keywords: agroecology / ecologically intensive agriculture / agroecosystem / agriculture / discipline

\section{Points importants}

Ce texte définit le concept d'intensification écologique appliquée à l'agriculture. Il revisite donc en partie les concepts de l'agronomie en les reprenant dans le cadre des concepts de l'écologie fonctionnelle. Mais les deux familles de concepts se rejoignent dans les raisonnements systémiques.

\section{Introduction}

La réflexion sur ce qui est appelé l'écologie intensive a commencé en France il y a 25 ans, en 1990, lors d'un séminaire de prospective animé par Thierry Gaudin ${ }^{1}$, auquel participait Hervé Bichat ${ }^{2}$, où nous avions proposé le concept de « jardin

\footnotetext{
*Correspondance : griffon.conseil@numericable.fr

${ }^{1}$ Ministère de la Recherche, directeur du Centre de prospective.

${ }^{2}$ Directeur général du CIRAD, Centre de coopération internationale en recherche agronomique pour le développement, animateur du Club 2100, « jardin planétaire ».
}

planétaire ». Il s'agissait, pour faire face à l'accroissement démographique planétaire et aux capacités limitées de la biosphère, de définir une agriculture qui soit plus productive mais aussi qui soit gérée sans atteintes à l'environnement et en conservant les qualités et capacités des sols pour les générations futures. N'ayant pas suscité d'intérêt pour les chercheurs, un autre concept lui a été substitué à partir de 1994, celui de "Révolution doublement verte ». Ce concept est apparu dans un groupe de travail du CGIAR $^{3}$ souhaité par Henri Carsalade ${ }^{4}$ et présidé par Gordon Conway ${ }^{5}$; nous y avions défini à nouveau les bases d'une agriculture à la fois plus productive que la « Révolution verte ${ }^{6}$ » et respectueuse de l'environnement. Mais c'est en 2007, lors du Grenelle de l'Environnement que le concept «d'Agriculture écologiquement intensive » est apparu. Par ce concept, nous définissions

\footnotetext{
${ }^{3}$ Groupe consultatif de la recherche agronomique internationale.

${ }^{4}$ Directeur général adjoint à la FAO.

${ }^{5}$ Écologue britannique.

${ }^{6}$ Nom donné à la politique agricole menée à partir du milieu des années 1960 en Inde, qui proposait l'utilisation de variétés à haut rendement de blé et de riz, l'utilisation d'engrais et une série de mesures d'appui, en particulier des subventions. Les hausses de rendement avaient été spectaculaires.
} 
une agriculture utilisant intensivement et en priorité les processus écologiques et biologiques, mais sans refuser les intrants conventionnels pourvu que leur usage soit subsidiaire et en cohérence avec la logique écologique et biologique présidant à l'évolution des systèmes productifs. Ce concept s'inscrit dans l'ensemble des idées rassemblées sous le nom d'agroécologie. L'agroécologie s'est déployée rapidement dans le monde, particulièrement dans les Amériques où le concept a sa propre histoire. En France, le CIRAD a très tôt promu les techniques agroécologiques, en particulier celles correspondant à « l'agriculture de conservation ». Un grand nombre de concepts voisins ont été promus dans différentes régions du monde. Toujours en France, l'agroécologie a fait, en 2015, l'objet d'une loi destinée à en assurer la promotion. L'espace des innovations que le concept d'intensification écologique ouvre semble important. Mais son cadrage théorique transdisciplinaire n'et pas simple. Cet article essaye de faire le point d'une manière sommaire sur les cadres théoriques de cette nouvelle « transdiscipline ».

De nombreux exemples concrets d'agroécologie existent dans le monde. Tous s'inspirent de l'écologie et plus précisément du fonctionnement des écosystèmes. On pourrait donc, pour la définir, se contenter de dire que l'agroécologie n'est rien d'autre que l'application de la science écologique aux écosystèmes agricoles que l'on qualifie dès lors « d'agroécosystèmes ». Mais la rencontre de l'écologie avec l'agronomie que le terme agroécologie annonce, et le grand nombre d'innovations que cette rencontre génère, méritent que l'on essaye d'en définir plus précisément les contours théoriques. Pour ce parcours théorique, il faut emprunter à plusieurs sciences et technologies, notamment, outre l'écologie, l'agronomie et la biologie, il faut solliciter la thermodynamique, la chimie et la science de systèmes. La réflexion agroécologique qui est abordée ici a surtout porté sur le concept d'intensification écologique qui exprime plus particulièrement le cœur du raisonnement de l'agroécologie vue sous son angle d'ingénierie productive. Mais, pour commencer, il faut clarifier un point : intensification et écologie sont deux termes qui semblent contradictoires. Est-ce si vrai ?

\section{2 Écologie intensive : oxymore ou terme scientifiquement légitime?}

Il faut donc expliquer le terme « écologiquement intensif» utilisé dans l'expression « agriculture écologiquement intensive $»$. Dès qu'il a été mis en circulation, beaucoup ont cru à une forme de provocation dans un contexte de débat social où associer le mot " intensif » au mot « écologie » paraissait vouloir marier l'eau et le feu ${ }^{7}$. Pour certains, il s'agissait d'une pure tromperie médiatique destinée à faire croire que l'agriculture intensive, réputée polluante, pouvait se réclamer de l'écologie. Pour d'autres, tout au contraire, cette association de mots en apparence contraires - un oxymore n'apparaissait pas aussi condamnable qu'on le voulait dès lors

\footnotetext{
${ }_{7}^{7}$ Le regretté Bernard Maris (assassiné dans les locaux de Charlie Hebdo en 2016) avait dit dans une chronique matinale radiodiffusée que intensif et écologie, c'était « le mariage de la carpe et du lapin ». Cette attitude méfiante a été surtout celle des courants écologistes.
}

qu'elle était porteuse de solutions nouvelles et réelles satisfaisantes au plan de l'environnement et de la productivité.

En fait, le mot « intensif » a été emprunté par le langage courant au langage des économistes. En économie agricole, on utilise toujours le mot intensif en l'associant à un intrant (au sens large c'est-à-dire facteur de production) ; on dira par exemple que l'agriculture de l'Inde est intensive en main d'œuvre car il faut beaucoup de main d'œuvre pour produire une certaine quantité de blé. Mais on dira que l'agriculture canadienne est intensive en capital car il faut beaucoup de capital technique pour produire une tonne du même blé. Utiliser le mot « intensif » appelle donc théoriquement à préciser le facteur de production qui est utilisé intensivement. Ce terme mesure donc le rapport entre une quantité d'intrant et la quantité d'extrant. De plus, on n'utilise ce concept que pour les systèmes productifs où l'on introduit beaucoup d'intrants. Dans le cas de l'agriculture conventionnelle, on devrait donc préciser qu'elle est intensive « en intrants », en l'occurrence, en engrais, en produits phytosanitaires et en capital. Cette définition de l'intensivité étant légitime, pourquoi dès lors, ne pas utiliser la formule «intensivité en écologie » si cela exprime le fait que l'on utilise intensivement des processus écologiques ? Ces processus écologiques sont des processus fonctionnels existant dans la nature. On peut les qualifier de « fonctionnalités ». L'agriculture écologiquement intensive est donc une agriculture intensive en fonctionnalités écologiques. Par exemple, on pourrait dire qu'une agriculture est intensive en photosynthèse dès lors que les capteurs solaires que sont les feuilles des plantes cultivées constituent une couverture du sol dense et permanente, et qu'il n'y a, dès lors, aucune perte dans l'utilisation des rayons du soleil car ceux-ci sont intégralement captés, ce qui n'est pas le cas des systèmes de culture où le sol est nu une partie importante du temps. De même, on pourrait parler d'une agriculture intensive en matière organique si l'on utilise beaucoup de compost, ou bien, pourquoi pas, intensive en auxiliaires des cultures si le système de culture utilise de nombreux ennemis des ravageurs présents.

Ceci étant précisé, il convient d'aller plus avant dans le concept d'intensification des fonctionnalités écologiques. D'abord, préciser les termes « écosystème, agroécosystème, anthropoécosystème ».

\section{Les écosystèmes productifs ; leur structure et leur fonctionnement}

La notion de système de production agricole date des années 1970 et marque l'entrée du langage de «l'analyse de système » dans l'agriculture. Les systèmes de production sont des systèmes associant le milieu à des ressources (sol, eau) et à des techniques. Ils concernent l'agriculture, l'élevage, l'arboriculture, la foresterie et l'aquaculture et, au sens large, les activées de cueillette, chasse et pêche. On pourrait les qualifier « d'écocultures » c'est-à-dire d'écosystèmes qui sont cultivés, c'est-à-dire modifiés dans le but de privilégier certaines cultures et certains animaux. Ce sont donc des écosystèmes, mais ils sont très particuliers : ils sont profondément transformés par les sociétés. On pourrait dire que ce sont des anthropoécosystèmes dans la mesure où c'est bien l'espèce humaine qui transforme massivement les écosystèmes, donc la biosphère dans sa quasi-totalité. Cela 
fait référence à l'émergence du terme « anthropocène » pour évoquer l'ère géologique actuelle où la présence transformatrice de notre espèce est très forte. Plus simplement, on utilise le terme « écosystème » pour les régions qui ne sont pas ou sont très peu pénétrées par les sociétés humaines, et le terme « agroécosystème » pour les écosystèmes modifiés par les sociétés pour la production agricole. Pour ces derniers, leurs transformations vont depuis quelques modifications (par exemple, une prairie naturelle dont on gère le broutage par des animaux domestiqués, ce qui est peu différent d'un parcours avec des animaux sauvages) jusqu'à de très grandes transformations (par exemple, la monoculture de maïs, des serres horticoles, des terrasses rizicoles à Bali... où l'écosystème initial est peu reconnaissable). Mais les agroécosystèmes restant fondamentalement des systèmes, ils gardent une structure écosystémique universelle qui associe un biotope et une biocénose. Exprimé autrement, un agroécosystème est un ensemble d'entités minérales et d'entités vivantes liées entre elles par des relations nombreuses et complexes. Ces entités sont des espèces biologiques domestiquées et sauvages, et des espèces physico-chimiques (des molécules du sol, par exemple), et les relations qui les lient sont des relations écologiques (prédation, consommation, concurrence...) ainsi que des relations chimiques (transformation des molécules, oxydation, réduction...), énergétiques et informationnelles.

Autre remarque importante, étant des systèmes, les agroécosystèmes sont dynamiques, c'est-à-dire qu'ils sont par nature en mouvement permanent et non en état figé et stable contrairement à ce que l'on pourrait croire lorsqu'on regarde un paysage immobile. Pour les décrire, il faut donc rendre compte de ce mouvement, ce qui n'est pas simple. S'il y a mouvement, c'est qu'il y a consommation et dépense d'énergie. Il faut donc représenter ce fonctionnement énergétique. Or, la représentation de la dynamique des agroécosystèmes en termes d'énergie n'est pas habituelle. Pourtant, elle est nécessaire et donc, la référence à la thermodynamique est pertinente.

En effet, les agroécosystèmes sont caractérisés par le fait qu'ils reçoivent de l'énergie solaire et qu'en présence de dioxyde de carbone, puisant par ailleurs des minéraux dans le sol, ils fabriquent des sucres. Ces sucres sont produits principalement dans les feuilles. Ces sucres sont des formes de stockage de l'énergie. C'est la fonction chlorophyllienne. Il existe d'autres formes moléculaires pour le stockage et le transport de l'énergie. Cette énergie « cristallisée 》 sous des formes d'énergie biologique et chimique est utilisée dans l'ensemble des structures des plantes pour permettre des transformations moléculaires. La photosynthèse permet donc aux organismes autotrophes que sont les végétaux d'accroître leur biomasse. Lorsque les conditions climatiques le permettent, cette croissance est permanente. Lorsqu'elles sont cycliques, la production est intermittente.

Les végétaux sont consommés par les hétérotrophes. Les sucres, mais aussi les lipides qui ont été biosynthétisés sont digérés. Ils apportent de l'énergie et des molécules qui sont métabolisées. Les hétérotrophes peuvent être consommés par des hétérotrophes de deuxième rang, puis de troisième rang formant des réseaux trophiques. Autotrophes et hétérotrophes finissent par mourir. Ils sont alors fragmentés et décomposés. Cette décomposition alimente le sol en molécules et en ions au bénéfice des végétaux. Au total, une grande partie de l'énergie est dissipée dans des relations diverses, mais il reste une partie d'énergie non utilisée qui se perd et quitte l'écosystème général : elle correspond à l'entropie.

L'ensemble d'un écosystème, et donc d'un agroécosystème, est donc alimenté par l'énergie solaire « à l'entrée » et perd donc de l'entropie " à la sortie ». Entre les deux extrémités, l'énergie est transportée et répartie dans le système. Elle alimente la constitution de molécules et alimente le métabolisme des plantes et des animaux. L'ensemble de ces plantes et de ces animaux (le réseau trophique) et l'ensemble de la " cascade énergétique » qui les lient peuvent être vus comme une structure dynamique. Cette structure étant vivante, elle est constituée d'entités qui se structurent et qui se déstructurent ; c'est donc une structure qui se renouvelle en permanence. On peut la qualifier de « structure dissipative ${ }^{8}$ » (Prigogine et Kondepudi, 1999 ; Roddier, 2012). Elle est très fluctuante dans son renouvellement. D'abord, elle est tributaire de la variation du flux solaire (variations nycthémérales et circadiennes, variations des saisons), et des variations de température et d'humidité. Elle est par ailleurs évolutive dans la mesure où la morphologie des écosystèmes change. Voir un agroécosystème dans la cadre de cette représentation, c'est concevoir son fonctionnement comme celui d'un système qui, d'une part crée de l'ordre et du désordre morphologique (évolution), et d'autre part dissipe de l'énergie dans son fonctionnement courant (métabolisme). Les conséquences de cette vision associant fonctionnement courant et évolution sont importantes pour la gestion. En effet, gérer un agroécosystème doit se faire selon une réflexion capable d'intégrer la notion de gestion de l'énergie en ayant en perspective la double préoccupation du renouvellement et de la trajectoire.

\section{La notion de fonctionnalité}

Décrire le fonctionnement d'un écosystème et donc d'un agroécosystème, c'est donc décrire d'une part ce que l'on pourrait appeler métaphoriquement le « tapis roulant métabolique », et décrire d'autre part les transformations de structure (trajectoire). Plusieurs approches sont possibles.

La première approche consiste à désigner les espèces qui sont concernées : espèces végétales, espèces animales, microorganismes, et décrire leurs relations. Les types de relations qui les lient sont nombreux : la relation proieprédateur, la relation hôte-parasite, le commensalisme ou l'amensalisme, le mutualisme ou le neutralisme, la symbiose, l'allélopathie ${ }^{9}$... Cette description constitue une très bonne entrée pour un repérage taxonomique. C'est la base de la représentation classique des écosystèmes en écologie fonctionnelle.

Pour aller plus loin dans la connaissance du fonctionnement, l'entrée moléculaire et chimique est indispensable. Chaque relation métabolique interne à un écosystème (c'est-àdire l'ensemble des entités qui le composent) est une relation

\footnotetext{
${ }^{8}$ Concept élaboré par Ilya Prigogine pour définir l'autoorganisation de structures dans un cadre thermodynamique. Voir (Prigogine et Kondepudi, 1999). L'application du concept au vivant vient de (Roddier, 2012).

${ }^{9}$ Propriété d'une plante de réduire ou de favoriser la germination ou le développement d'une plante voisine.
} 
qui est une transformation moléculaire. Elle se décrit sous la forme d'une équation chimique résumant une réaction (ou une cascade de réactions) c'est-à-dire la transformation des molécules avec consommation ou émission d'énergie généralement en présence de catalyseurs. Chaque équation décrit par une égalité la conservation de la matière (les atomes). L'ensemble enchaîné des équations représente le métabolisme d'une entité appartenant à un écosystème, et l'ensemble des équations de l'ensemble des entités de l'écosystème représente le métabolisme complet de l'écosystème. Cette vision représente donc un écosystème comme un système unifié d'équations chimiques transcendant les entités internes existantes. Cela amène à considérer chaque écosystème comme un superorganisme et donc à respecter autant que possible l'intégrité des écosystèmes et agroécosystèmes existants ${ }^{10}$ en tant que construction originale. Cette conception devrait pouvoir permettre dans l'avenir, lorsqu'elle sera opérationnelle, de faciliter l'évaluation ex ante des effets d'innovations chimiques dans un écosystème tout entier.

Une autre approche du fonctionnement écosystémique est permise par la connaissance des gènes de l'ensemble des êtres vivants d'un écosystème appelé métagénome. Là aussi, on conçoit l'écosystème comme un organisme complet. Le concept est opérationnalisé : on dispose du métagénome de compartiments d'écosystèmes comme un sol. Chacun des gènes de l'écosystème est à l'origine d'une succession d'évènements métaboliques (réactions chimiques) qui caractérise une voie métabolique. La séquence de ces évènements se répète d'une part en raison de l'initialisation par les gènes, puis d'autre part en raison de la répétition à chaque stade de la séquence des conditions de réalisation des réactions (présence des molécules-ressources, présence des catalyseurs, conditions nécessaires de milieu...). Les gènes initialisent donc les réactions. Les séquences de ces réactions se déroulent ensuite jusqu'à un stade où il y a stabilisation. Une fonctionnalité peut donc être décrite comme un ensemble de gènes et de voies métaboliques définissant une cohérence fonctionnelle d'ensemble, par exemple, l'ensemble métagénome et métamétabolome caractérisant l'humification d'un sol d'un écosystème donné. Le métagénome est la mémoire d'ensemble des mécanismes d'initialisation des séquences d'évènements métaboliques d'un écosystème. Le transcriptome est l'ensemble des ARN messagers correspondant aux gènes qui ont été exprimés. Le métabolome est l'ensemble des molécules intermédiaires, hormones, métabolites secondaires, molécules signal... qui interviennent dans le déroulement des voies métaboliques. Le génome, le transcriptome et le métabolome peuvent être vus comme des bases de données rassemblant l'information relative aux différents processus métaboliques d'un écosystème vu comme un super ou métaorganisme. Cette information est contenue dans toutes les structures moléculaires concernées. Cette information transcende aussi les entités existantes d'un écosystème ou d'un agroécosystème : les co-évolutions, par exemple, donnent lieu à des informations qui sont complémentaires et distribuées entre les espèces coévoluantes. Se référer à cette vision en termes d'information

\footnotetext{
${ }^{10} \mathrm{C}$ 'est ce qu'a fait la Convention sur la diversité biologique en reconnaissant les écosystèmes comme des constructions à prendre en compte tout autant que les espèces.
}

devrait permettre de cartographier les sous-ensembles de génome qu'il pourrait être utile de modifier pour adapter rapidement un écosystème à des évolutions de milieu ; par exemple, il serait utile de connaître dans un agroécosystème, l'ensemble des gènes et des voies métaboliques de résistance à la sécheresse afin de cibler les transformations pertinentes à opérer afin de faciliter une adaptation d'un agroécosystème basée sur la modification génétique.

La représentation d'un agroécosystème peut donc être plurielle (fonctionnelle, chimique, génétique et informationnelle...). Dans tous les cas, l'ensemble des structures et des processus dynamiques concernés est si vaste et si complexe qu'un agroécosystème n'est pas intellectuellement facilement manipulable. Or nous avons besoin de manipuler les agroécosystèmes avec aisance pour les adapter stratégiquement à nos besoins. Il faut donc trouver une voie de simplification de la représentation de leur complexité. La voie dite de « simplexité ${ }^{11}$ » (Berthoz, 2009) s'impose alors. Cette voie consiste dans le cas des écosystèmes, à définir des entités fonctionnelles intermédiaires, c'est-à-dire des clusters manipulables. Le concept qui en résulte est celui de fonctionnalité (noté Fnlt). Une fonctionnalité écosystémique est un ensemble de relations répétées et enchaînées définissant un sousensemble d'un écosystème. C'est donc un ensemble répétitif de fonctions. Il est délimité par une entrée et une sortie. La photosynthèse, par exemple, est une fonctionnalité. L'humification de la biomasse dans un sol en est une autre. L'inhibition locale du développement d'un végétal par la présence d'un autre végétal ou allélopathie négative est un autre cas.

L'ensemble des fonctionnalités peut être structuré en trois niveaux de généralités correspondant à des échelles différentes. Au niveau le plus fin se situent les fonctions (notées $F n$ ). À un niveau agrégé se situent des "Méta-fonctionnalités 》 (notées $M F n l t$ ). Par exemple, la métafonctionnalité «Fertilité du sol » comprend la fonctionnalité « Fixation symbiotique de l'azote par les légumineuses », et celle-ci comprend la fonction « Luzerne-stockage de l'azote », la fonction « Trèfle », etc. On peut aussi imaginer un niveau plus agrégé en rejoignant la notion de compartiment d'un écosystème. On peut aussi différencier les fonctionnalités écosystémiques et les fonctionnalités internes aux différentes espèces, ou fonctionnalités physiologiques.

\section{L’agroécologie : gérer un agroécosystème qui est toujours en déséquilibre}

Généralement, l'objectif principal de la gestion d'un agroécosystème est d'obtenir le meilleur rendement possible $e^{12}$ tout en cherchant à " conserver ses équilibres » selon l'expression souvent utilisée. Le terme « équilibre » est ici plus l'expression d'une métaphore qu'une expression décrivant un phénomène réel. Le terme « équilibre » est souvent utilisé car il est toujours vu comme un phénomène positif et,

\footnotetext{
${ }^{11}$ La simplexité est une manière de rendre simple et compréhensible une entité complexe. Le concept a été créé par Alain Berthoz.

${ }^{12}$ D'autres objectifs sont possibles comme améliorer la qualité des produits, ou améliorer la viabilité et la résilience des agroécosystèmes.
} 
symétriquement, le déséquilibre est toujours interprété comme un phénomène négatif. Mais qu'est-ce qu'un équilibre dans un écosystème?

Tout d'abord, s'il s'agit bien d'équilibres, il ne peut s'agir que d'équilibres dynamiques puisqu'un écosystème est par nature en mouvement permanent. Cela entraîne que la notion d'équilibre dynamique pur et parfait décrirait un état d'un système qui serait caractérisé par des mouvements immuablement réguliers, de telle sorte que son image dynamique serait identique à ce qui résulterait d'un « arrêt sur image ». Ce serait donc, pour un écosystème, un état identique à lui-même mais sans cesse renouvelé. Dans la réalité, sans être totalement impossibles, tels états ne peuvent être que des cas limite, des exceptions de courte durée.

Dans la nature, on observe, au contraire, que les écosystèmes connaissent des variations permanentes. Les variables de commande externes comme, par exemple, les variables climatiques (facteurs abiotiques) sont sans cesse en mouvement : l'intermittence des flux solaires, la variation des températures, du degré d'humidité... Quant aux variables internes, elles sont mises en mouvement par les variables externes et ainsi que par le jeu des interactions internes; elles sont donc, elles aussi, en mouvement perpétuel et irrégulier. Les agroécosystèmes sont de la même manière en changement permanent, en plus, en raison des pratiques agricoles qui se succèdent pendant un cycle végétatif (les itinéraires techniques). On peut considérer qu'il en va de même pour les fonctionnalités : l'ensemble des fonctionnalités d'un agroécosystème ne connaît pas d'équilibre. Dès lors, faut-il qualifier de déséquilibre la dynamique qui résulte de ces variations ?

Le jeu interne des fonctionnalités d'un écosystème est complexe. Deux fonctionnalités peuvent être reliées par des relations de formes diverses comme peuvent être liées entre elles des variables fonctionnelles en termes mathématiques. On peut faire l'hypothèse que les fonctionnalités sont assimilables à des variables au sens de l'analyse de système, et sont liées par des fonctions. Par exemple, l'effectif d'une population d'une proie est lié à l'effectif de la population du prédateur. Autre exemple : la quantité d'humus disponible dans une parcelle est liée au volume des résidus de culture de l'année antérieure. Ces fonctions sont quelquefois simples (des phénomènes additifs ou soustractifs, des fonctions linéaires). Elles sont très souvent complexes. Par exemple la relation proie-prédateur qu'il faut savoir gérer dans les pratiques de lutte biologique prend la forme d'une équation du second degré qui, au premier abord est simple $: f(x)=4 p x(1-x) ; x \in(0,1)$ où $x$ est le nombre de prédateurs d'une année donnée, $f(x)$ est le nombre de prédateurs l'année qui suit, et $p$ le rapport de reproduction en effectifs des prédateurs par rapport aux proies. En prenant des exemples numériques, on trouve que le nombre de prédateurs l'année $n+1$ est lié au nombre de prédateurs de l'année $n$ selon une courbe en forme parabolique. Si on itère le calcul en utilisant le même modèle, on peut obtenir une courbe en « double bosse ». Pour certaines valeurs, l'itération peut se stabiliser sur un point remarquable où le nombre de prédateurs et de proies sont constants d'une année à l'autre ; c'est un « point attractif » qui fait penser à un équilibre dynamique stable et qui est un point singulier. Pour d'autres valeurs du paramètre $p$, la courbe ressemble à des montagnes russes. Un calcul où l'on accroît le nombre des itérations (ce qui ressemble à la réalité) montre que la valeur des points attractifs peut évoluer vers une situation chaotique (Boudine, 2016). Les relations de concurrence entre deux populations obéissent donc à des lois dont les applications numériques concrètes peuvent se révéler surprenantes. Mais il ne s'agit là seulement que d'un simple exemple de relations. Au sein d'un agroécosystème, les relations sont très nombreuses, enchaînées et interactives, ce qui crée des situations à d'autres niveaux de complexité. La réalité peut donc comporter dans une même représentation des comportements complexes de relations élémentaires avec des comportements complexes de systèmes eux-mêmes complexes : des relations de concurrence en réseau, et des relations de synergie, des évolutions de morphologie par apparition de conditions nouvelles... On est donc obligé de conclure à ce stade à une très grande difficulté de calculabilité concrète des états prévisionnels d'un agroécosystème. On peut aussi conclure que vraisemblablement, dans la très grande majorité des cas, un écosystème, et donc un agroécosystème, est en déséquilibre permanent, mais qu'il n'est pas impossible que localement et sur de courtes périodes, il puisse y avoir des points attractifs correspondant à des états dynamiques stables.

Autre élément de complexité : la dynamique morphologique des systèmes. À un instant donné, un écosystème a une forme particulière, par exemple, pour un biome terrestre local donné, il y a (en simplifiant) un stock de sol utile, un stock de nutriments, un ensemble d'espèces végétales, animales et microbiennes, des espèces cultivées choisies, un stock de graines d'adventices, un ensemble d'espèces constituant la faune de la strate épigée... Entre tous ces éléments internes à un écosystème existe un ensemble complexe de relations à effet synergique ou antagoniste. Cet ensemble a de nombreux états particuliers possibles selon les valeurs prises par les variables internes, au sein d'une même architecture du système. Cet ensemble peut connaître des évolutions sans changer cette architecture. Il peut donc changer d'état au sein d'une même forme. Un exemple illustratif bien que peu probable aide à se représenter cette pluralité des états dans le cadre d'une même forme : dans un agroécosystème, les effectifs de telle espèce d'adventices peuvent augmenter pendant un petit nombre d'années, ce qui change l'état du système, mais le nombre des autres espèces peut rester le même, ce qui ne fait pas varier la morphologie. Mais il se peut que certaines variables atteignent des valeurs qui entraînent un basculement vers un changement de forme. Par exemple, la baisse continue de la matière organique d'un sol peut se traduire par des changements lents d'état, puis brutalement par un effondrement structural du sol qui est un changement de la morphologie du système. Il y a donc des évolutions internes qui peuvent être formulées comme des lois de discontinuité du genre «si, alors » : « si une fonctionnalité Fnltx atteint une valeur limite $x_{l}$, alors le système change de forme ». Tout système peut donc évoluer et changer de forme selon des lois de morphogenèse ou de morpho-destruction préexistantes. Cela crée donc des discontinuités et des bifurcations dans l'évolution du système.

Ces changements morphologiques peuvent s'enchaîner. Il peut donc en résulter des dynamiques enchaînées négatives. Par exemple, une succession de niveaux de tassement de sols de plus en plus importants, puis un épisode d'érosion peuvent être le résultat d'une perte linéaire (dans le temps) de matière organique puis une catastrophe érosive (rupture dans la structure du sol, discontinuité). Une accélération d'enchaînements négatifs peut entraîner des « avalanches » d'évènements 
morphologiques, par exemple, en suivant le même cas, une érosion répétée sur un sol tassé. On peut même avoir jusqu'à un « effondrement » en cas de rupture de plus grande ampleur, par exemple un glissement de terrain en cas de gros orage.

Inversement, il peut y avoir des processus enchaînés d'aggradation. Un agroécosystème peut être géré de telle manière qu'il améliore différentes fonctionnalités dans un cadre morphologique inchangé, ou si se créent des fonctionnalités nouvelles dans le cadre de changements morphologiques améliorants. En reprenant le même exemple dans une version inverse, une amélioration lente et continue de la teneur en matière organique d'un sol sahélien peut se traduire par le retour d'un fonctionnement biologique plus intense - comme l'indique quelquefois le retour des termites - se traduisant par une évolution morphologique positive.

Dégradation et aggradation peuvent aussi se succéder. Une dégradation morphologique d'un agroécosystème se traduit par une moins bonne utilisation de l'énergie, car les structures dissipatives sont loin d'être à leur maximum de constitution. L'entropie augmentera. Au contraire, si une aggradation succède, elle se situera dans un champ de reconstitution de structures dissipatives et l'entropie diminuera.

Au total, un écosystème ou un agroécosystème est toujours en déséquilibre. Il peut s'agir de déséquilibres induits par le jeu naturel des variables externes. Il y a de fortes chances dès lors que les déséquilibres soient limités en amplitude. Ils restent alors inscrits dans le champ de viabilité du système c'est-à-dire l'ensemble des morphologies et des états qui ne connaissent pas de dégradation. Si la gestion du système conduit à l'affaiblir continûment, ou si survient un accident dû au comportement erratique d'une variable externe (par exemple, une période de basse température, des intempéries catastrophiques), le système peut alors bifurquer négativement. Il n'y a vraisemblablement aucune raison qu'un écosystème connaisse une attraction vers des situations d'équilibre dynamique. Il peut néanmoins exister des mécanismes intégrés de stabilisation, comme par exemple l'homéostasie chez un animal ou l'autorégulation de la température interne. Mais il n'y a pas semble-t-il de mécanisme équivalent dans les écosystèmes. Un agroécosystème soumis à des variations naturelles de contexte et à une gestion tâtonnante risque donc en permanence d'emprunter des trajectoires non viables. Il doit donc être piloté avec prudence de manière à ne pas sortir de son cadre de viabilité. Ce principe de pilotage est un principe de sauvegarde des agroécosystèmes.

\section{La viabilité d'un agroécosystème}

S'il y a très peu de raisons dans des conditions d'environnement habituelles, qu'un écosystème fluctuant dans un espace de variation borné, connaisse des situations d'attraction vers un point d'équilibre, il n'y a vraisemblablement qu'aussi peu de raisons qu'il dérive naturellement vers des situations de dégradation. La convergence inévitable vers un état attracteur, tout comme la divergence spontanée du système ne sont sans doute pas des situations nominales. Mais un agroécosystème, à la différence d'un écosystème peut dériver. L'intervention de l'homme peut en effet produire des erreurs durables, comme l'épuisement lent de la fertilité d'un sol. Sa gestion peut induire, comme on l'a vu, des évolutions chaotiques déstabilisatrices ou des cascades d'évolution morphologique négatives.

Chaque fluctuation qui sort du cadre habituel de fluctuation peut induire une trajectoire de dégradation. Or, tout agroécosystème, dans un statut morphologique donné, peut avoir de nombreux états internes possibles, certains étant proches des limites de viabilité. Par exemple, soit un agroécosystème de monoculture maïsicole (ce qui définit un type de morphologie) ; il peut avoir de nombreux états chacun étant défini par les conditions du milieu et par divers itinéraires techniques comme plus ou moins d'irrigation, le recours appuyé ou allégé aux herbicides, le recours à la lutte biologique ou aux insecticides pour lutter contre les chenilles de la pyrale... Tous ces états sont viables c'est-à-dire susceptibles d'évoluer en restant dans le cadre de viabilité défini par la morphologie de l'agroécosystème. Mais s'il y a dérive et dégradation de l'état de l'agroécosystème avec un cadre morphologique inchangé, par exemple en laissant le système être envahi par des pyrales par un mauvais contrôle, son état risque de se rapprocher des frontières de viabilité de la morphologie dans laquelle il évolue. Si les frontières de viabilité sont franchies, le système change alors de morphologie, donc de dynamique, voire même, on peut dire qu'il peut aller jusqu'à « s'effondrer » c'est-à-dire connaître des dégâts très étendus dus à l'invasion par le ravageur. Tout doit donc être fait pour surveiller la trajectoire d'un agroécosystème afin éventuellement de la corriger, et d'éviter d'entrer dans des trajectoires de dégradation morphologique.

En résumé, un agroécosystème peut donc avoir plusieurs statuts morphologiques et pour chacun de ces statuts, il peut avoir des états internes plus ou moins viables. L'ensemble des statuts morphologiques peut être ordonné selon sa richesse en termes de structures dissipatives, depuis des morphologies très dégradées jusqu'à un ensemble de statuts et d'états se situant vers un maximum. Une morphologie dégradée peut être, par exemple toujours en maïsiculture, caractérisée par de l'érosion, par des sols envahis d'adventices, par des pullulations fréquentes d'insectes et par des traitements phytosanitaires nombreux. À l'inverse, une morphologie et des états très satisfaisants pourront être proche d'un climax, c'est-à-dire d'un maximum de biomasse. Toujours pour un agroécosystème maïsicole, un climax serait par exemple caractérisé par un couvert végétal maximal, un sol stabilisé et riche en stock de fertilité, et une autorégulation des pullulations d'insectes. Le but d'une agriculture écologiquement intensive sera donc d'évoluer en restant dans son cadre de viabilité et d'y être présent dans des conditions les plus proches du climax.

\section{Intensifier les fonctionnalités : l'amplification}

Si le but de la gestion d'un agroécosystème est de se situer le plus possible dans une situation de climax, chaque fonctionnalité d'un agroécosystème, dès lors qu'elle est utile, doit prendre des valeurs élevées. Si elle est jugée comme ayant des effets négatifs, elle doit prendre des valeurs faibles. Or chaque fonctionnalité peut prendre des valeurs différentes, plus ou moins grandes. Par exemple, on cherchera à maximiser le taux de matière organique. Mais, à l'inverse, le stock de 
graines d'une espèce d'adventices dans un sol pouvant être plus ou mois grand, ce qui déterminera le potentiel invasif de cette espèce, on cherchera à le diminuer le plus possible. Autre exemple, si la surface d'un sol est couverte par des légumineuses associées à la culture principale, la densité des légumineuses déterminera le potentiel de production de nitrate produit par la fixation symbiotique de l'azote par les légumineuses. Chaque fonctionnalité peut donc être considérée comme une variable quantifiable du système.

Ces fonctionnalités - ou variables du système - sont liées entre elles par des relations d'influence. Par exemple, plus la densité de couverture du sol par des légumineuses est élevée, plus les légumineuses concurrencent certaines adventices. L'ensemble des fonctionnalités et des relations qui les relient décrit donc le fonctionnement du système dans sa totalité. Ainsi, quand on amplifie la valeur d'une fonctionnalité qui a des effets positifs, cette amplification a des effets d'amplification répercutés sur le système dans son ensemble. Cette répercussion suit les chemins d'influence définis par la nature des relations qui existent entre les variables. Par exemple, l'association d'une légumineuse de couverture à une espèce cultivée augmente la quantité d'azote disponible et favorise la croissance de l'espèce cultivée. Il y aura donc un effet positif sur le volume de la biomasse totale, puis sur celle des résidus de culture disponibles, ce qui pourra accroître en conséquence le volume de l'humus, lequel aura à son tour d'autres effets en répercussion.

Les variables et les relations qui les relient peuvent être représentées par un diagramme sagittal : les variables sont reliées par des flèches qui tracent l'influence d'une variable sur une autre. C'est une représentation de la structure et de son fonctionnement qui est éloquente (car pédagogique), mais quelquefois difficile à réaliser concrètement. On peut aussi représenter ces relations d'influence par une matrice carrée mettant en relation toutes les variables du système entre elles. C'est une représentation tout aussi rigoureuse. Cette matrice carrée de dimension $A(n, n)$, notée $A=\left\{a_{i, j}^{n}\right\}$ est telle que lorsqu'il existe une influence d'une variable $i$ sur une autre $j$, alors $a_{i, j}=1$, et lorsqu'il n'y a pas d'influence, $a_{i, j}=0$. Des valeurs 1 qui sont voisines dans la matrice définissent des chemins d'influence. En élevant en puissance la matrice carrée, on obtient une matrice stable au bout d'un certain nombre d'itérations. Cette matrice a alors épuisé toutes les possibilités d'emprunts des chemins d'influence, y compris les bouclages de rétroaction positive. Elle figure alors un « idéal » d'amélioration défini uniquement par les enchaînements positifs. On peut aussi essayer de doter la matrice de valeurs numériques de manière à essayer de représenter aussi bien les effets positifs d'amplification que les effets négatifs de réduction. On a alors le moyen de simuler des trajectoires.

Ainsi, d'un point de vue théorique, on sait représenter l'ensemble des effets de répercussion de l'amplification d'une fonctionnalité sur un agroécosystème ${ }^{13}$. Selon les chemins d'influence il peut $y$ avoir des différences importantes d'efficacité de l'effet d'amplification. D'abord, une même variable peut avoir différentes influences sur différentes variables. Par exemple, toujours avec des légumineuses, une couverture végétale pourra avoir des conséquences positives

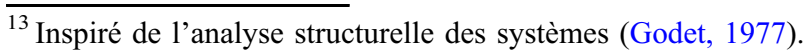

sur la fertilité azotée, sur le rendement de la plante cultivée principale, mais aussi sur le maintien d'un bon degré d'hygrométrie dans la partie superficielle du sol, sur le maintien d'une température utile au même endroit, et sur la protection du sol contre l'érosion. Ces différents effets peuvent à leur tour avoir des effets que l'on dira indirects par exemple, les effets de l'amplification du rendement sur la biomasse retournant au sol. Puis des effets de troisième rang comme la contribution de cette biomasse à la production de matière organique, etc. La succession définit des chemins d'influence. Dans ce cas, la variable ayant diverses influences et des effets enchaînés aura une efficacité remarquable. C'est le cas des couvertures végétales dans les agroécosystèmes.

Dans d'autres cas, diverses variables ont des effets positifs convergents sur une autre variable. Par exemple, l'humidité d'un sol provenant de couvertures végétales, la présence de racines ayant un effet de détassement et l'amélioration des conditions d'environnement pour les lombrics sont des variables qui convergent vers l'amélioration de la structure du sol. Dans ce cas, la variable qui reçoit diverses influences positives aura aussi un rôle remarquable.

Dans d'autres cas encore, il peut y avoir des effets de feedback. Le bouclage d'un chemin d'influence positive qui se répercute en effet de deuxième rang, puis de troisième, puis de rangs ultérieurs avec retour vers la variable initiale crée un phénomène de « cercle vertueux ». Par exemple, l'accumulation de fertilité dans un sol résultant de la restitution des résidus de culture, de la non-perturbation de la biologie du sol par un labour, et de la présence de légumineuses, se traduit à son tour par une meilleure production de biomasse, laquelle pourra entraîner une plus grande restitution au sol des résidus de culture... De tels bouclages positifs de rétroaction définissent en théorie des mécanismes à effets sans fin, mais qui sont dans la réalité toujours limités. En effet, il y a rapidement des ressources limitantes qui stabilisent le processus; ici les limites climatiques (température, lumière solaire), ou les limites de nutriments du sol.

La conjonction de ces différentes configurations positives conséquences multiples, convergence de multiples effets, boucles de rétroaction - définit des synergies. Ces synergies peuvent être mises à profit dans la gestion des agroécosystèmes. Elles peuvent être activées en agissant préférentiellement sur les variables clés c'est-à-dire se situant à des nœuds d'influence ou dans des situations de bouclage. Ainsi, l'amélioration de la structure et de la fertilité d'un sol par des couvertures végétales apparaît comme centrale (un nœud d'influence) pour améliorer une grande partie du fonctionnement d'un agroécosystème. Cette synergie est celle qui est principalement opérationnalisée dans l'agriculture de conservation. De même, la réintroduction de l'élevage dans un système céréalier permet l'amélioration de la fertilité par les apports de lisiers et fumiers et d'azote par les légumineuses, ce qui tend à accroître la biomasse et son retour dans les sols... Cette synergie est celle qui lie l'agriculture et l'élevage.

\section{Atteindre un climax éco-technologique ?}

Les écosystèmes et les agroécosystèmes ne sont pas constitués que de relations d'influence positives. Il existe aussi des relations d'influence négatives. Par exemple, l'explosion 
épidémique de certaines maladies dans des troupeaux entraînant la mort. Dans ce cas, un « envahisseur biologique » détruit massivement une partie importante de l'agroécosystème. Dans la plupart des cas, selon le volume de telle ou telle variable influence, il peut y avoir des effets induits positifs ou négatifs. Par exemple, un excès d'azote peut entraîner une sensibilité des plantes aux maladies cryptogamiques et aux parasites, en même temps qu'il risque d'entraîner des pollutions des masses d'eau. Á l'inverse, un défaut d'azote entraîne un défaut de croissance et un jaunissement des feuilles aboutissant à un faible rendement. Toute amplification se fait généralement dans des bornes de variation qui correspondent à des limites du domaine de viabilité. La gestion des agroécosystèmes doit donc assurer à la fois l'amplification des fonctionnalités afin d'obtenir de meilleurs rendements. Elle va donc s'éloigner des situations de défaut, mais aussi s'éloigner des situations d'excès lorsque celles-ci peuvent entraîner des "effets externes négatifs " 14 . De même, elle doit contrôler les fonctionnalités négatives.

Cela revient à trouver par la gestion du système un état ou un ensemble d'états correspondant à un maximum viable à des performances élevées (et restant donc viables). Atteindre ces états revient à choisir les trajectoires les plus appropriées. Ainsi, un écosystème est à tout moment dans une trajectoire où le rayonnement solaire constitue le moteur de la production de biomasse. Sa croissance est contrainte par les limites propres aux autres ressources que sont l'eau, les nutriments du sol, la dynamique des maladies et ravageurs des autotrophes... Le maximum qui peut être atteint constitue un climax - point ultime d'un mécanisme de croissance -, concept qui est purement théorique car il semble définir un état dynamique de stabilité, alors que le système est toujours instable.

Par ailleurs, dans un agroécosystème, gérer les fonctionnalités ne consiste pas seulement à manipuler celles qui sont naturelles. Il y a, en effet, non seulement les fonctionnalités naturelles, mais aussi des fonctionnalités artificielles c'est-àdire créées par les sociétés. Ce sont des techniques et plus généralement la technologie. Elles comprennent deux ingrédients : une intervention fonctionnelle dans l'agroécosystème, et du travail humain qui la met en œuvre. Par exemple, l'arrachage d'une adventice comprend l'intervention dans la concurrence phytosociologique (concurrence entre plantes), et le travail mécanique d'arrachage qui est utilisateur d'énergie. Dans la pratique, la technologie est toujours associée à des fonctionnalités naturelles qu'elles modifient. Dans le même exemple, c'est bien une technologie - l'arrachage, le binage (qu'il soit manuel, réalisé avec une traction animale attelée ou un tracteur) qui s'insère dans le jeu de la concurrence entre espèces végétales. Avec l'évolution technologique, les agroécosystèmes sont de plus en plus constitués de techniques, donc de fonctionnalités artificielles, mais les fonctionnalités naturelles restent le fondement du fonctionnement des agroécosystèmes. Aussi, la recherche d'un état (ou d'un ensemble d'états) viable et tendant vers le maximum de production de biomasse est une recherche d'un climax sensu lato que l'on peut qualifier de " climax éco-technologique ».

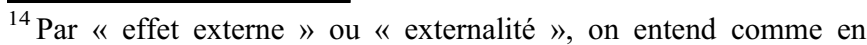
économie, un effet non attendu ou non prise en compte a priori dans un raisonnement.
}

\section{Intensifier les fonctionnalités par la résilience}

Gérer un agroécosystème de manière à ce qu'il soit viable, n'est pas simplement chercher à l'amplifier et à valoriser les effets de synergie. C'est aussi chercher à limiter les chocs et les effets des chocs. Pour cela, il y a dans la nature plusieurs fonctionnalités possibles : la dissuasion, l'avertissement et la préparation au choc, le bouclier, l'endurance, la duplication, et la réparation. La dissuasion contre les attaques est à la fois ancienne et moderne : par exemple, utilisation d'épouvantails à oiseaux dans le passé aujourd'hui, installation de perchoirs pour les rapaces afin d'éloigner d'autres oiseaux qui sont des ravageurs. La notion d'avertissement existe chez les plantes en cas d'agression, par l'émission de molécules signal qui sont reconnues par les plantes voisines au sein desquelles se déclenchent des mécanismes d'émission de toxines qui éloignent les agresseurs. Certaines plantes produisent en permanence des molécules qui éloignent les agresseurs ce qui constitue un mécanisme " bouclier » qui rend ces plantes pratiquement inaltérables et leur confère un avantage compétitif dans l'espace naturel. Ce sont des familles généralement originales avec peu d'espèces et qui ont singulièrement un caractère envahisseur. La duplication des fonctionnalités ou redondance fonctionnelle est un mécanisme rare : une fonctionnalité hors d'état de fonctionner est remplacée par une autre agissant en parallèle. En écologie, il s'agit généralement d'espèces dont les niches écologiques sont proches ; si l'une diminue, l'autre s'y substitue. Enfin, la réparation est la capacité de reconstruire des structures biologiques et écologiques si celles-ci ont été amputées. Cela peut être le cas par exemple pour des écosystèmes agroforestiers où l'on pratique l'abattis-brûlis en rotation longue ; les clairières agricoles sont reconquises par les espèces forestières voisines pour reconstituer, en quelques décennies, un paysage équivalent à l'antérieur. Ce processus naturel peut être consolidé par des actions complémentaires des sociétés comme des replantations. La résilience peut donc prendre divers aspects.

Mais le mécanisme de résilience le plus courant est celui de la constitution de stocks systémiques. Son caractère générique est vraisemblablement dû au fait qu'un écosystème est en perpétuelle variation et qu'il doit lui-même s'adapter à cette variation. Dans un écosystème, chaque espèce a des mécanismes de survie pour faire face aux situations momentanées de défaut. Les végétaux accumulent des réserves d'amidon dans les graines, les tiges ou les racines. Il s'agit de réserves de glucides ou de lipides, ou bien encore d'eau. La mise à disposition des réserves se fait soit dans le cadre d'une reprise de cycle végétatif (sortie de dormance), soit dans le cadre d'un mécanisme de reproduction (pour la germination d'une graine).

Dans un agroécosystème, la résistance aux chocs peut donc se faire en cherchant systématiquement à constituer des réserves de facteurs de production indispensables. C'est le cas par exemple pour la conservation des eaux du sol de toutes les manières possibles, ou le stockage du carbone dans les sols en vue de lutter contre l'effet de serre, ou encore en vue de constituer un stock de matière organique utile à la croissance de la plante cultivée. 
Une autre forme de résilience est conférée par la diversité et la complexité de l'architecture des agroécosystèmes. Plus un système est complexe, plus ses variations sont susceptibles d'être autolimitées. Dans un modèle proie-prédateur à seulement deux agents, on peut par itération aboutir à des situations de chaos ou d'état dynamique stable, comme on l'a vu. Dans un modèle où il y a un grand nombre de proies et prédateurs conjugués, la probabilité d'émergence de pullulations envahissantes est faible car la probabilité d'être combattue par d'autres auxiliaires est forte. Avec plus de diversité, il y a émergence de phénomènes d'autolimitation des amplitudes de fluctuation des espèces car tout commencement de pullulation est susceptible de rencontrer un mécanisme trophique adverse. Ainsi, une monoculture sur de grandes surfaces entraîne toujours des pullulations de ravageurs et l'installation d'un auxiliaire est utile mais difficile à conduire car il faut maîtriser les calendriers végétatifs relatifs de la proie et de l'auxiliaire. Au contraire, des dispositifs de polyculture en mosaïque et des cultures associées en mélange, le tout en prenant soin d'associer en plus des plantes qui sont des habitats pour un grand nombre d'auxiliaires, sont des dispositifs où toute tentative de pullulation ou presque à une probabilité élevée de se heurter à la « contre-attaque » d'un des auxiliaires. C'est donc la diversité et corrélativement (dans ce cas) la complexité des systèmes trophiques qui permettent la résilience.

\section{Piloter la viabilité et l'optimalité}

Toute la connaissance systémique qui vient d'être évoquée sert à piloter la viabilité et obtenir les performances souhaitées. Piloter la viabilité d'un agroécosystème, c'est donc d'une part amplifier les fonctionnalités positives sous des conditions de dosage (prise en compte des bornes de viabilité), et d'autre part construire des mécanismes de résilience. Ces deux familles de mesures ne sont pas faciles à appliquer.

Amplifier les fonctionnalités positives exige une vraie dextérité. Il s'agit en effet, comme on l'a vu, d'éviter tout d'abord les excès comme les défauts dans les variables de commande ou les variables d'influence. Il faut notamment calculer les effets multiplicateurs inattendus, par exemple un faible apport d'engrais azoté mais qui, ajouté à des apports dus à des légumineuses et un stockage dans le sol, aboutit à des excédents importants d'azote dans des nappes phréatiques par migration.

Il s'agit ensuite de maintenir les amplifications de différentes fonctionnalités dans un cadre de cohérence qui est celui de la viabilité et celui de l'efficience. À l'extrême, un apport élevé d'un facteur de production isolé aboutit, faute de cohérence, au forçage du système par ce facteur. Les forçages portent le risque de déformer le système et de produire des effets externes négatifs. Par exemple, un apport intensif d'aliments énergétiques à des vaches laitières, avec modification rapide de cette alimentation et de sa composition, a vraisemblablement des effets favorables au développement de mammites. La suralimentation des animaux d'élevage à croissance accélérée peut entraîner des maladies métaboliques et une composition nutritionnelle des viandes défavorable à la santé humaine.

Il y a donc un espace de viabilité (des valeurs bornées) pour effectuer le réglage des variables utilisées pour agir sur les agroécosystèmes. Les frontières de viabilité sont généralement floues et instables, chaque valeur étant généralement tributaire d'autres. Il y a donc pour tout agroécosystème une «périphérie » floue et instable proche des frontières de viabilité. Il y a symétriquement un espace de sûreté pour la viabilité qui correspond à la minimisation d'un maximum de risques. Cela définit au total une performance qui peut apparaître dans certains cas comme modeste, mais avec une résilience confortable, surtout si elle est consolidée par une stratégie de maximisation des stocks de viabilité. L'optimum durable est donc difficile à trouver. Pour l'approcher, il s'agit d'abord d'opter pour un niveau donné de résilience (ou de son contraire, le risque), ce qui est un choix individuel ; certains ont une forte aversion pour le risque, d'autres non. Ensuite, il faut choisir dans un ensemble de statuts morphologiques possibles ceux qui sont souhaitables, ce qui revient concrètement à choisir les rotations de cultures ainsi que les assolements et associations d'espèces, et choisir les éventuelles productions d'élevage et les liens entre les activités d'agriculture et d'élevage. Puis, en fonction de l'état présent de l'agroécosystème, définir une transition morphologique et une conduite dans le réglage des différentes variables. Le mode de raisonnement qui permet une telle optimisation est vraisemblablement un algorithme par tâtonnement qui explore les solutions possibles et permet d'identifier les plus souhaitables.

\section{La part du naturel et la part des technologies nouvelles : la bio-inspiration}

L'agriculture écologiquement intensive a été conçue dans la perspective de substituer le plus possible des procédés naturels à des intrants chimiques entraînant des risques pour l'environnement et pour la santé humaine. Mais, elle ne s'interdit pas d'utiliser ces intrants par pragmatisme, ou dans des phases de transition, toujours avec modération. Il n'y a aucune raison de limiter l'espace des technologies à l'utilisation des seules fonctionnalités naturelles et à pratiquer une agroécologie que l'on pourrait qualifier de purement « naturelle ». L'Agriculture écologiquement intensive ne peut être que favorable à des solutions technologiques nouvelles, pas seulement écologiques ou biologiques mais qui n'entraînent pas d'effets externes négatifs. Ces solutions seraient en grande partie mimétiques de fonctionnalités existant dans la nature, ou bien bio-inspirées, c'est-à-dire imitant les fonctionnalités naturelles. Cela concerne beaucoup les molécules chimiques de défense des cultures, les médicaments, ou des molécules intervenant dans la gestion physico-chimique des sols. Ce choix de bio-mimétisme et de bio-inspiration présente l'intérêt que les innovations proposées s'inscrivent dans le cadre des fonctionnements naturels et donc qu'ils peuvent s'y insérer en théorie sans risques majeurs, dans certaines limites de viabilité mais ce n'est pas parce qu'une molécule est naturelle qu'elle ne présenterait pas de dangers, notamment en fonction des doses pratiquées. Ce choix paraît cependant moins risqué que celui qui a longtemps présidé à la recherche phytosanitaire, celui de l'exploration dans de nombreuses directions des possibilités de toxicité chimique sans assurance de pouvoir envisager l'ensemble des risques encourus.

Le champ de l'imitation des phénomènes biologiques et écologiques est très important. Il y a là un gisement 
d'opportunités. Par exemple, l'allélopathie adverse (l'effet de toxicité d'une plante sur une plante voisine) recèle des mécanismes potentiellement intéressants pour maîtriser les adventices. La recherche indienne a établi des listes de plantes à capacité allélopatique et leurs cibles en vue de pouvoir jongler d'une manière systématique et complète avec elles pour que les successions culturales réduisent durablement les adventices. C'est un peu ce qui se faisait en Europe autrefois mais de manière moins systématique avec les cultures dites « nettoyantes » comme l'avoine. Mais connaître les mécanismes chimiques et les imiter aurait une toute autre portée. De la même manière que pour l'allélopathie, l'inventivité des industries des polymères et des bio-matériaux peut déboucher par bio-inspiration sur des molécules extraordinairement fixatrices d'eau et peut être des ions dans des solutions captives, ce qui résoudrait potentiellement les problèmes de lixiviation dans les sols. L'utilisation de gènes issus d'une espèce - qui correspondent à des fonctionnalités naturelles - que l'on transfère dans une autre espèce est une autre forme d'intensification écologique. La synthèse de polymères biostructurés de plus en plus complexes, la synthèse de briques moléculaires de synthèse par des transformations chimiques progressives, et l'autoassemblage d'objets moléculaires sont des voies qui laissent espérer des innovations importantes.

L'amélioration génétique de certaines fonctionnalités peut aussi être envisagée. Les techniques de remplacement " gène pour gène " avec une précision complète ouvrent des perspectives nouvelles. Récemment, par exemple, il est apparu possible d'améliorer le rendement photosynthétique des plantes en limitant le processus de résistance de ces plantes aux dommages éventuels induits par l'ensoleillement, mécanisme qui réduit l'efficacité de la photosynthèse (Kromdijk et al., 2016). Ce mécanisme pouvant être maitrisé, il y a une possibilité d'intensifier la photosynthèse. D'autres possibilités s'ouvriront vraisemblablement pour améliorer la biologie des sols ou limiter la consommation du phosphore dont le stock mondial est limité.

\section{L'intervention à bonne échelle d'espace et de temps par les STIC}

L'écologie, comme l'agronomie est une science de la localisation. Tout phénomène écologique se réalise dans un cadre spatial et selon un cadre de durée qui sont donnés. Par exemple, l'espace de la rhizosphère créé par une graine a son originalité bactérienne propre. Le mycélium de champignon que l'on trouve dans une parcelle et qui n'est souvent qu'un seul et même individu de plusieurs tonnes de biomasse définit une fonctionnalité qui permet le transit et la redistribution des éléments minéraux à l'échelle de la parcelle. L'eau qui circule dans une parcelle dépend de l'hydrosystème à l'amont dans le bassin versant. À chaque phénomène correspond une échelle d'espace : rhizosphère, parcelle, bassin versant... À chaque phénomène correspond aussi une échelle de temps, par exemple le temps épidémiologique pour des attaques de ravageurs, le temps du rythme phénologique des plantes. Ces échelles introduisent des contraintes de gestion de l'agroécosystème : un agriculteur ne peut pas surveiller efficacement en permanence toute la superficie des ses parcelles ; un éleveur ne peut pas non plus suivre chacun de ses animaux à partir d'une certaine taille de troupeau.

Les sciences et techniques de l'information et de la communication (STIC) apportent une solution pour travailler efficacement aux différentes échelles. Ces STIC associent différentes technologies. D'abord celle des capteurs d'information : images de parcelles provenant de satellites ou de drones, images biologiques d'animaux, coefficients biologiques d'animaux... Ces capteurs démultiplient les observations des agriculteurs et des éleveurs. Des logiciels leur fournissent très rapidement des informations critiques par les traitements qui résultent de ces informations (attaques prévisionnelles de ravageurs, épidémies dans les troupeaux) qu'il s'agisse de prévision et d'avertissement ou de faits observés. Ces données sont aussi regroupées par les constructeurs de capteurs pour bâtir de gigantesques bases de données dont le traitement pourrait faire émerger des solutions par définition insoupçonnées. Des logiciels systèmes experts peuvent aussi proposer des solutions issues de l'expérience acquise (traitements phytosanitaires, régulation des hydrosystèmes, régime spécifique pour des lots d'animaux...). Enfin, des robots peuvent exécuter des décisions issues de ces logiciels d'analyse et d'expertise : traitement microlocalisé par des drones de maladies en émergence, affouragement spécifique des vaches laitières selon leur état biologique...

La précision, mais plus largement, l'analyse et l'intervention multi-échelles et multi-opportunités changent radicalement la capacité d'intervention sur les agroécosystèmes. Ces interventions démultiplient les capacités de vigilance. Elles permettent d'envisager des automatismes décisionnels pour les phénomènes les plus fréquents et banaux (comme l'adaptation des régimes alimentaires des animaux), ce qui introduit des fonctionnalités technologiques automatiques. Elles facilitent aussi de la flexibilité, par exemple pour choisir entre un désherbage mécanique ou calorique robotisé et un traitement chimique ou encore une succession culturale nettoyante. Il y a donc convergence entre les besoins de l'écologie intensive et les STIC.

\section{Conclusion : des développements futurs}

Ce tour d'horizon rapide n'a pas d'ambition théorique nouvelle. La seule ambition a été, depuis l'origine de cette réflexion, de mieux faire rencontrer les approches de la réalité issues de l'écologie fonctionnelle et de l'agronomie. Comme on l'a vu, pour comprendre et gérer les agroécosystèmes, il est nécessaire d'embarquer d'autres disciplines dans les raisonnements et particulièrement la systémique, tandis que la thermodynamique offre un cadre de rationalisation nouveau. Cela crée des conditions nouvelles pour l'acquisition des compétences des chercheurs travaillant sur les agroécosystèmes et sur leurs gestionnaires que sont les agriculteurs. La transdisciplinarité devient donc indispensable pour comprendre les agroécosystèmes. Il apparaît clairement par ailleurs, à la lumière de ces différentes considérations, qu'un des enjeux importants pour l'avenir est l'apprentissage de la gestion systémique. 


\section{Références}

Berthoz A. 2009. La simplexité. Odile Jacob, coll. Sciences. EA N13: 9782738121691.

Boudine J-P. 2016. Proies et prédateurs : pourquoi de tels écarts ? QR\#HS1-S\&V. 19-26.

Godet M. 1977. Crise de la prévision, essor de la prospective. Paris : PUF.

Kromdijk J, Glowacka K, Leonelli L, et al. 2016. Improving photosynthesis and crop productivity. Science 354 (6314): 857861.

Prigogine I, Kondepudi D. 1999. Thermodynamique : des moteurs thermiques aux structures dissipatives. Éditions Odile Jacob, coll. Sciences.

Roddier F. 2012. Thermodynamique de l'évolution. Parole Éd., Artignosc.

\section{Pour aller plus loin}

Altieri MA. 2013. L'agroécologie. Condé sur Noireau : Charles Corlet.
Barbault R. 1997. Écologie génétale. Paris : Dunod.

Barbault R, Weber J. 2010. La vie, quelle entreprise. Paris : SeuilScience ouverte.

Burel F, Baudry J. 2006. Écologie du paysage. Paris : Lavoisier. Tec\&Doc.

Doré T, Le Bail M, Martin P, Ney B, Roger-Estrade J, Sebillotte M. 2006. L'agronomie aujourd'hui. Paris : QUAE éd. Coll. Synthèses. Frontier S, Pichod-Vialle D. 1990. Écosystèmes. Paris : Masson. Gliessman SR. 2015. Agroecology, 3rd ed. Boca Raton : CRC Press. Griffon M. 2013. Qu'est-ce que l'agriculture écologiquement intensive ? Paris : QUAE éd., AEI.

Lefeuvre JC. 2013. Les invasions biologiques. Paris : Buchet Chastel. Lefroy EC, Hobbs RJ. 1998. Agriculture as a mimic of natural ecosystems. Dordrecht: Wer Academic Publishers.

Lévêque C. 2001. Écologie. Paris : Dunod.

Matagne P. 2002. L'écologie et son histoire. Paris : Delachaux et Niestlé.

McNeely J, Sher S. 2003. Ecoagriculture. Washington: Island Press. Thomas F, Archambeaud M. 2013. Les couverts végétaux. Paris : Éditions France Agricole.

Wojtkowski PA. 2006. Introduction to agroecology. New York: FPP.

Citation de l'article : Griffon M. 2017. Éléments théoriques en agroécologie : l'intensivité écologique. OCL 24(3): D302. 